\title{
Integrated Channel Selector for Directing Fluid Flow Using Thermoreversible Gelation Controlled by a Digital Mirror Device
}

\author{
Yoshitaka Shirasaki, ${ }^{1,2}$ Masayasu Tatsuoka, ${ }^{2}$ Mai Yamagishi, ${ }^{1}$ Jun Mizuno, ${ }^{2}$ \\ Shuichi Shoji, ${ }^{2}$ and Takashi Funatsu ${ }^{3}$ \\ ${ }^{1}$ Reserch Center for Allergy and Immunology, RIKEN, 1-7-22 Suehiro-cho, Tsurumi-ku, Yokohama, \\ Kanagawa 230-0045, Japan \\ ${ }^{2}$ Graduate School of Advanced Science and Engineering, Waseda University, 3-4-1 Ohkubo, Tokyo 169-8555, Japan \\ ${ }^{3}$ Laboratory of Bioanalytical Chemistry, Tokyo University, 7-3-1 Hongo, Tokyo 113-0033, Japan
}

Correspondence should be addressed to Takashi Funatsu; funatsu@mail.ecc.u-tokyo.ac.jp

Received 6 October 2012; Revised 11 February 2013; Accepted 19 February 2013

Academic Editor: Takahiro Arakawa

Copyright (c) 2013 Yoshitaka Shirasaki et al. This is an open access article distributed under the Creative Commons Attribution License, which permits unrestricted use, distribution, and reproduction in any medium, provided the original work is properly cited.

An integrated channel selector system employing thermoreversible gelation of a polymer was developed. Here, we show a system with $3 \times 3$ arrayed microchannels having nine crossing points. Infrared laser irradiation was used to form gel areas at several crossing points in arranging a flow path from the inlet to one of the nine outlets passing through certain junctions and channels. The multipoint irradiation by the infrared laser was realized using a personal-computer-controlled digital mirror device. The system was demonstrated to be able to direct flow to all nine outlets. Finally, we achieved to produce flexible paths for flowing particles including side trips.

\section{Introduction}

Microfluidic technologies are recognized as powerful tools in many fields such as chemistry, biology, and medicine [1-3]. These technologies make it possible, for example, to analyze a tiny amount of samples and allow the development of quitehomogeneous reactor systems called microprocess servers. In the microfluidic systems that have been developed, fluids are usually moved in one direction through prebuilt channels, which provide only limited flexibility. To increase flexibility and complexity in changing flow direction, numerous valve systems controlled by various forces have been developed, for example, a pneumatically controlled valve constructed of soft material, miniaturized mechanical valves fabricated using micromachining technologies, and regulation of flow resistance changes in a microchannel employing $\mathrm{pH}$-sensitive or temperature-sensitive gelation or light-driven wettability changes [4-12]. Among these valves, the pneumatically controlled valve has been applied in many types of devices because of its simplicity of fabrication and the possibility of multiple-valve manipulation $[4,7]$. However, the operation of the pneumatic valve is controlled by an off-chip solenoid valve, and the large-scale integration of microvalves requires the same numbers of off-chip control solenoid valves and world-to-chip connectors [13]. We have developed flow control systems that have one input and two to five outputs thermoreversible gelation of hydrogel with infrared (IR) laser-induced local heating [14-16]. We succeeded to direct the flow selectively in a noncontact way, but the techniques based on the flow switching at only one junction. Here, we extend the flow control mechanism to the flow control on a flexible pathway junction with independently regulated 18 valves.

\section{Materials and Methods}

2.1. Structure of a Microchannel Matrix. We designed an integrated channel selector as a matrix of microchannels having one inlet and nine outlets as shown in Figure 1(a). 


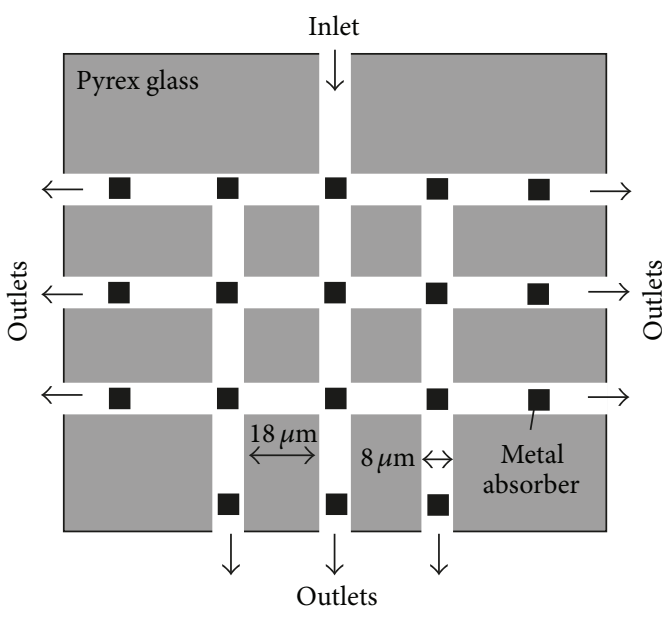

(a)

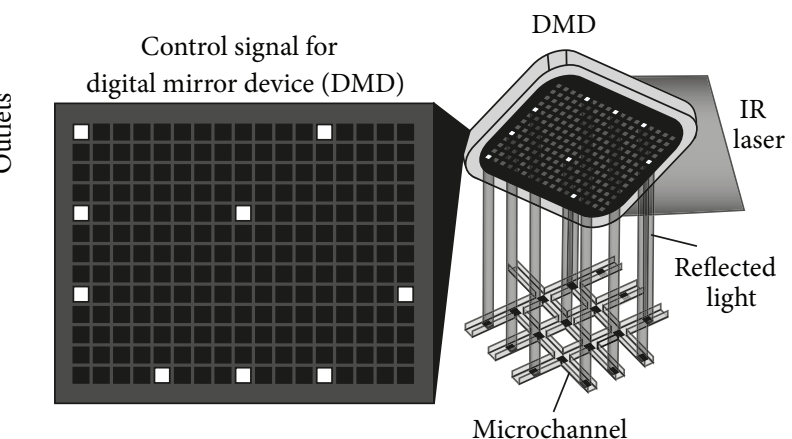

(b)

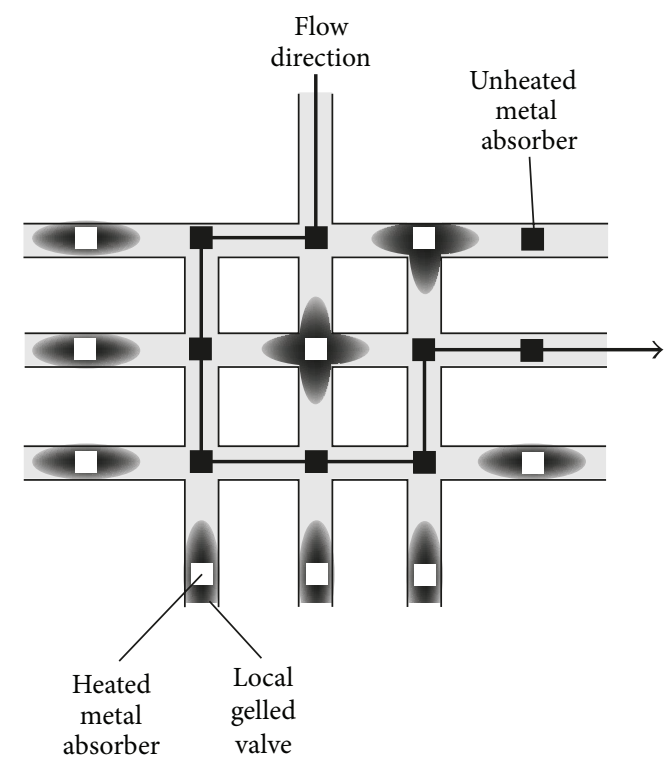

(c)

FIGURE 1: Operating principle of the channel selector system. (a) Layout of the microchannels used in this study. (b) Multipoint heating system with IR irradiation using a DMD. (c) Principle for regulation of the flow direction.

To realize efficient heating, $\mathrm{Cr} / \mathrm{Pt}$ thin metal absorbers were formed at every crossing point of flow paths and the points just before the respective nine outlets.

\subsection{Principle of Multiple Flow Control. We utilized thermal} reversible gelation polymer solution for flow controlling in a microchannel. In this work, Mebiol gel, which is a block copolymer of poly (N-isopropylacrylamide-co-n-butyl methacrylate) and poly (ethylene glycol), was used [15]. The polymer in liquid form (sol state) at room temperature turns into an elastic hydrogel (gel state) above the critical temperature $\left(36^{\circ} \mathrm{C}\right)$. Viscosity of gel state has about 1,000 times higher than that of sol state, and locally formed gel is able to plug flow in a microchannel. Pluronic F-127 also has similar rheological change and was successfully used in some fluid controlling technique $[8,9,11]$, though the thermal control for gelation of the polymer should be strict because of its secondary gelto-sol transition at higher temperature [17]. Mebiol gel, which has no thermal hysteresis and maintains gelled structure at higher temperature, has an advantage in flow control. In previous work, we achieved continuous plugging on bifurcating channel with $1 \mathrm{~mm} \mathrm{sec}^{-1}$ flow velocity, keeping fast Open/Close responses [15] with single focused infrared laser. Here, we extend the heating spot with metal absorber and multispot illumination system by a digital mirror device (DMD) (Figure 1(b)). Carrier solution of Mebiol Gel in sol state with microbeads was introduced into the inlet. After the target microbead was detected, a certain set of metal absorbers was heated by IR-laser-beam irradiation reflected by the personal-computer-controlled DMD, which created the flow path from the inlet to the specific outlet (Figure 1(b)). Carrier solution at irradiated points transformed into the gel state, which plugged microchannels and changed the 


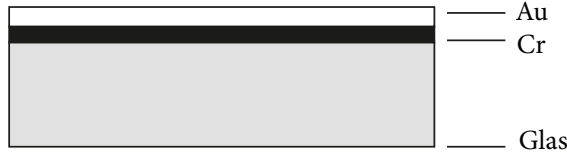

(a) $\mathrm{Cr} / \mathrm{Au}$ depositing

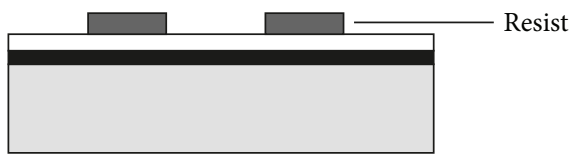

(b) Resist patterning

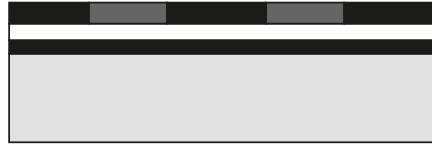

(c) Ni plating

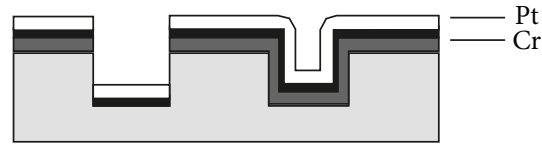

(f) $\mathrm{Cr} / \mathrm{Pt}$ spattering

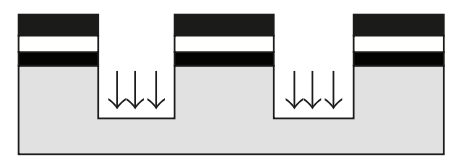

(d) Glass etching

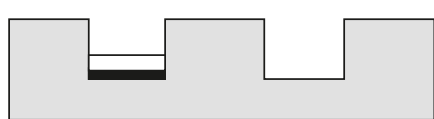

(g) Liftoff

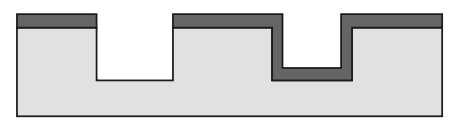

(e) Resist patterning

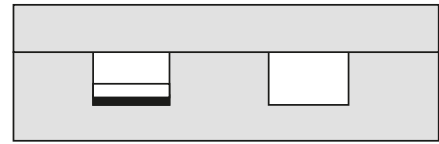

(h) Glass bonding

FIGURE 2: Fabrication of the channel selector system.

direction of flow (Figure 1(c)). The extent of gelation could control by changing the inputted power of IR laser to the metal absorbers.

\subsection{Fabrication of the Channel Selector System. Figure 2} shows the fabrication of the channel selector device. First, $\mathrm{Cr}$ and $\mathrm{Au}$ metals were sequentially deposited on $500 \mu \mathrm{m}$ thick Pyrex glass substrate with layer thicknesses of $30 \mathrm{~nm}$ and $40 \mathrm{~nm}$, respectively (Figure 2(a)). Au film was used as a seed layer for electrolytic plating. A positive photoresist (TSMRV90 27 cp, Tokyo Ohka Kogyo Co., Ltd.) was coated on the Au film, and the pattern of the microchannel matrix was transferred by photolithography (Figure 2(b)). A Ni layer, serving as a mask for inductively coupled plasma reactive-ion etching (ICP-RIE), was deposited with a layer thickness of $700 \mathrm{~nm}$ by electrolytic plating (Figure 2(c)). Microchannels $5 \mu \mathrm{m}$ deep were etched in the Pyrex glass substrate by ICPRIE, and all metals on the Pyrex glass were then removed (Figure 2(d)). Another positive photoresist (PMER P-LA900, Tokyo Ohka Kogyo Co., Ltd.) was coated on the Pyrex glass and transferred the pattern of heat absorbers (Figure 2(e)). $\mathrm{Cr}$ and $\mathrm{Pt}$ were sequentially deposited with layer thicknesses of $70 \mathrm{~nm}$ and $80 \mathrm{~nm}$, respectively, by sputtering (Figure 2(f)). After removing the photoresist (Figure 2(g)), Pyrex glass substrate was thermally bonded to the Pyrex glass cover plate with thickness of $170 \mu \mathrm{m}$ at $560^{\circ} \mathrm{C}$ (Figure $2(\mathrm{~h})$ ). We optimized thickness and heat active condition of $\mathrm{Cr} / \mathrm{Pt}$ absorber. The absorber could absorb 97\% of energy of inputted IR laser. To maintain a constant temperature for the device, the top of the device was attached to a water heat sink.

2.4. Optical Setup. Figure 3 shows the optical system for fluorescence detection and IR laser irradiation. An inverted optical microscope (IX71, Olympus Optical, Tokyo, Japan) equipped with an objective lens (UApo/340 20x; Olympus Optical) was used. A 30-W IR laser (808 nm, LIMO32-F200DL808-LM, LIMO Lissotschenko Mikrooptik GmbH) was used for the IR source. The IR laser light incident on the
DMD (removed from DLP projector, V-807, PLUS vision Co.) was reflected by a subset of micromirrors, which were in the on state, directly through the objective lens to irradiate microchannels while by the other micromirrors, which were in the off state, directed to an optical trap. Reflected IR laser was introduced to the channel selector via a relay lens, a dichroic mirror (a custom-made $505 \mathrm{~nm}$ long path filter; Asahi Spectra Co., Ltd), and an objective lens. The power of IR laser at the focal plane was $2 \mathrm{~W}$ when IR laser was reflected by all the micromirrors on DMD. A change in the angle of micromirrors was triggered by target signals processed and sent by a personal computer. Rhodamine B, which was mixed at a trace amount in carrier solution to visualize the microchannels, and microbeads (FluoSpheres Polystyrene Microspheres, $1.0 \mu \mathrm{m}$, yellow-green fluorescent, for tracer studies; Molecular Probes) were excited by a mercury lamp via an excitation filter (D540/25x; Chroma Technology, Co.) and dichroic mirror (Q565LP; Chroma Technology, Co.). Acquired fluorescence was cast on a charge-coupled device (CCD) camera (CCD-300RCX; DAGE-MTI) through an emission filter (D605/55 m; Chroma Technology Co.). Image signals from the CCD camera were converted by an image acquisition board (GINGA+; Linx Corp.) and analyzed using Halcon software (Linx Corp.). The DMD was controlled by a laboratory-made program written in Visual Basic.

\section{Results and Discussions}

3.1. Computational Fluid Dynamics Analysis. To search for appropriate conditions for sol-gel transition in the channel selector, computational fluid dynamics (CFD) analysis was carried out. From the CFD analysis, critical parameters were found. The width of the microchannel should be $8 \mu \mathrm{m}$, because if it was less, the flow resistance was too high, and if more, heat diffusion through the microchannels was too fast. Furthermore, the interval of the microchannel lattice should be at least $20 \mu \mathrm{m}$ because if less than that, applied heat accumulates within the microchannel lattice. 


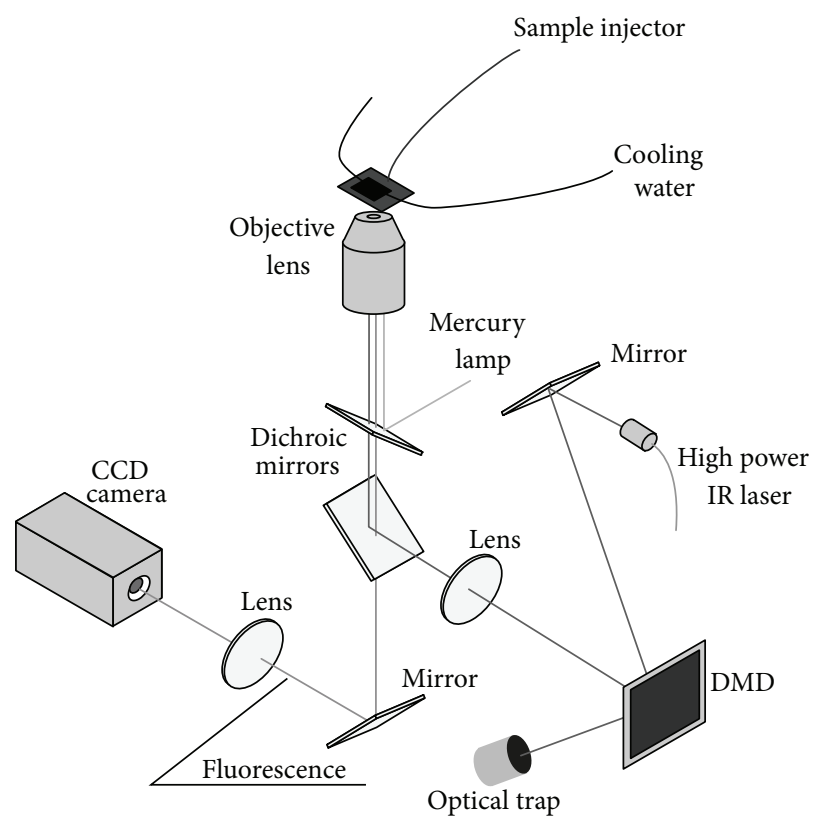

FigURE 3: Schematic diagram of the optical setup for the channel selector system.

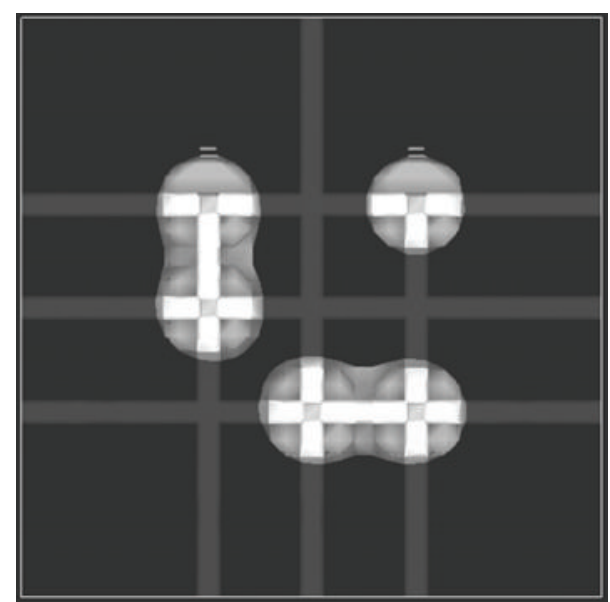

FIGURE 4: CFD analysis of temperature distribution in the microchannel. The channel had $8 \mu \mathrm{m}$ width and $26 \mu \mathrm{m}$ channel length between intersections. Highlighted area indicates the area over $30^{\circ} \mathrm{C}$.

Additionally, the channel selector device should be made of Pyrex glass rather than silicone because of the small thermal conductivity of Pyrex glass. A simulation of the application of thermal energy to the set of metal absorbers under the above condition showed that the temperature distribution was sufficiently limited to produce the correct flow path (Figure 4). The required power of the IR laser was estimated as $100 \mathrm{~W} \mathrm{~m}^{-2}$ from the simulation results.

3.2. Demonstrations of Channel Selection. A mixture of Mebiol Gel, Rhodamine B, and fluorescent microbeads was introduced to the microchannel through the inlet with a constant-pressure pump at $0.15 \mathrm{MPa}$; the flow velocity was about $500 \mu \mathrm{m} \mathrm{sec}^{-1}$. First, we tried to move the target microbeads to each outlet. Uniform square-shaped illumination could form the flow path to each outlet in independent trials but failed in switching the flow path. The error often occurred when the path was switched to another more distant outlet. This phenomenon could be explained by difference in flow resistance to different outlets. We found that more distant outlets had higher flow resistance and needed more increased resistance to stop flow. Therefore, we optimized input power of IR laser to each absorber by changing the polygonal areas through a trial and error process. In this process, we also found that accumulation of heat in the chip also affected the control of flow. Minimization of total input power to the chip was performed. Fluorescence images in Figure 5 show the trajectories of fluorescent beads flowing toward different outlets under the heating of specific points as indicated in the illustration next to the fluorescence images. Each flow path was successfully controlled so that beads moved to the specific outlet. Sequential switching of flow path demonstrated with switching speed was less than $300 \mathrm{~ms}$. Flow rate of beads was reduced to $97 \%$ on average through the inlet to outlets. This result showed leak-less flow control by heat-induced local gelation, showing that high-responsive flow path selection was realized.

Next, we tried to make a more flexible flow path to ensure the expected performance not only near the inlet/outlet but at the center of the lattice. In this trial, the shortest paths were not taken. Figure 6 shows an example of such a demonstration. For this trajectory, all the heated points functioned as barriers against the sample flow.

\section{Conclusion}

We developed a one-inlet, nine-outlet integrated channel selector with a flexible pathway junction system employing 


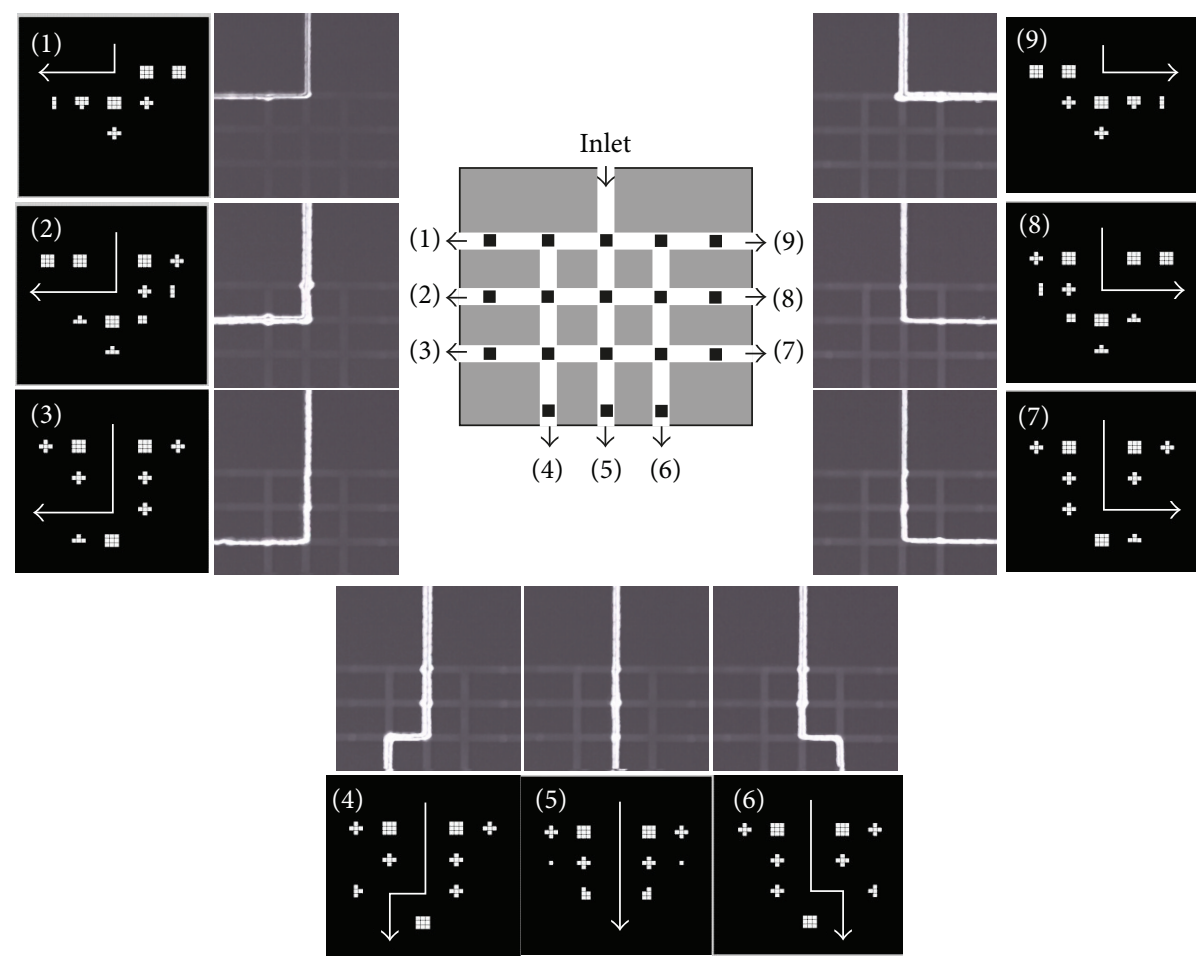

Figure 5: Flow control to select the route from the inlet to each specific outlet. Nine sets of the fluorescent image and an illustration of the irradiation situation are shown. Numbers in illustrations correspond to the numbers of outlets as shown in the central figure. To move fluorescent microbeads to the specific outlets (as indicated by an arrow in each illustration), IR laser light was irradiated as indicated by the white polygonal patterns. Each polygon is a combination of one-nine squares, indicating that the irradiation beam at that point was reflected by one-nine micromirrors. Each microbeads trajectory follows the expected route.

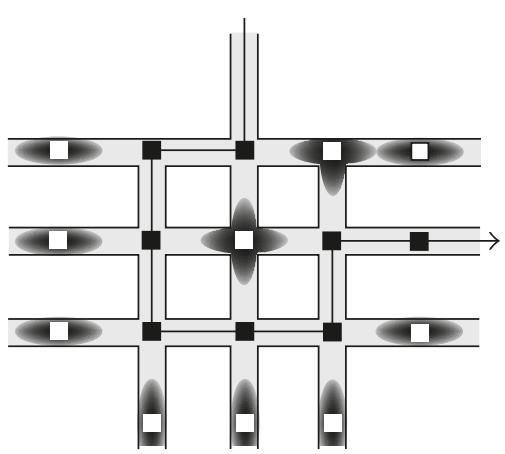

(a)

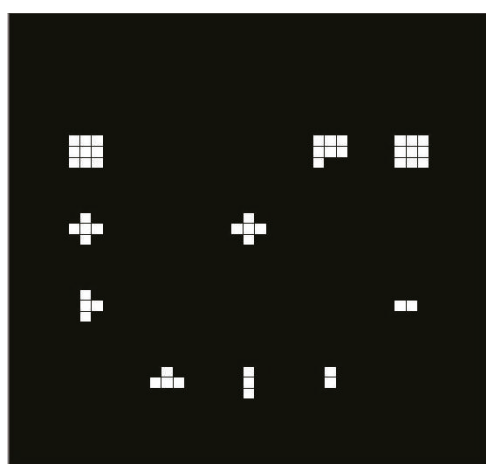

(b)

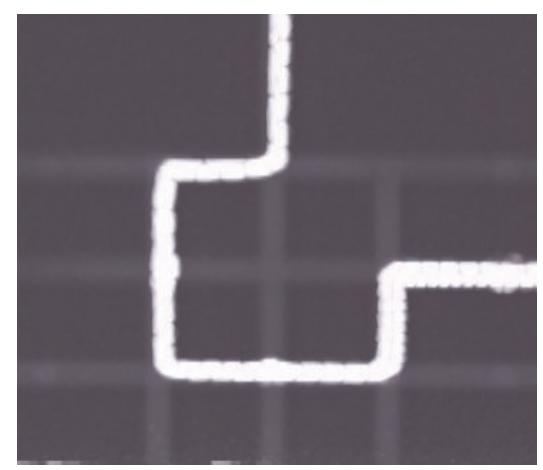

(c)

Figure 6: Flow control for a more flexible route from the inlet to an outlet. (a) The expected route (arrow) and required gelation points (darkened areas). (b) The IR laser irradiation pattern represented as white polygons. Each polygon is a combination of two-nine squares, representing that the irradiation beam at that point was reflected by two-nine micromirrors. (c) Fluorescence image of the trajectory of the fluorescent microbead indicating that heat-induced gelation worked well to block and change the direction of fluid flow at each point.

the thermoreversible gelation of Mebiol Gel and IR irradiation controlled by a DMD. This system controlled 18 valves independently through noncontact energy transmission. This operating principle enables us to integrate all machinery for a channel selector within a $100 \mu \mathrm{m}$ square and thus offers new fluid systems such as a demultiplexer that is applicable to micrototal analysis systems.

\section{Acknowledgments}

The authors thank Shota Watabe and Tomohiro Shimomae for experimental support, as well as members of Funatsu laboratory and Shoji laboratory for discussions. This research was partly supported by SENTAN, JST, and by Grant-in-Aid for Scientific Research (S), Grant no. 23226010, JSPS of Japan. 


\section{References}

[1] C. Aoyama, A. Saeki, M. Noguchi et al., "Use of folded micromachined pillar array column with low-dispersion turns for pressure-driven liquid chromatography," Analytical Chemistry, vol. 82, no. 4, pp. 1420-1426, 2010.

[2] Y. Xu, K. Jang, T. Yamashita, Y. Tanaka, K. Mawatari, and T. Kitamori, "Microchip-based cellular biochemical systems for practical applications and fundamental research: from microfluidics to nanofluidics," Analytical and Bioanalytical Chemistry, vol. 402, pp. 99-107, 2012.

[3] Y. Song, M. Noguchi, K. Takatsuki et al., "Integration of pillar array columns into a gradient elution system for pressuredriven liquid chromatography," Analytical Chemistry, vol. 84, pp. 4739-4745, 2012.

[4] I. E. Araci and S. R. Quake, "Microfluidic very large scale integration (mVLSI) with integrated micromechanical valves," Lab on a Chip, vol. 12, pp. 2803-2806, 2012.

[5] T. Arakawa, T. Sameshima, Y. Sato et al., "Rapid multi-reagents exchange TIRFM microfluidic system for single biomolecular imaging," Sensors and Actuators B, vol. 128, no. 1, pp. 218-225, 2007.

[6] G. Chen, F. Svec, and D. R. Knapp, "Light-actuated high pressure-resisting microvalve for on-chip flow control based on thermo-responsive nanostructured polymer," Lab on a Chip, vol. 8, no. 7, pp. 1198-1204, 2008.

[7] J. W. Hong and S. R. Quake, "Integrated nanoliter systems," Nature Biotechnology, vol. 21, no. 10, pp. 1179-1183, 2003.

[8] M. Krishnan and D. Erickson, "Optically induced microfluidic reconfiguration," Lab on a Chip, vol. 12, pp. 613-621, 2012.

[9] M. Krishnan, J. Park, and D. Erickson, "Optothermorheological flow manipulation," Optics Letters, vol. 34, no. 13, pp. 1976-1978, 2009.

[10] D. S. Reichmuth, T. J. Shepodd, and B. J. Kirby, "On-chip highpressure picoliter injector for pressure-driven flow through porous media," Analytical Chemistry, vol. 76, no. 17, pp. 50635068, 2004.

[11] B. Stoeber, C. M. J. Hu, D. Liepmann, and S. J. Muller, "Passive flow control in microdevices using thermally responsive polymer solutions," Physics of Fluids, vol. 18, no. 5, Article ID 053103, 2006.

[12] G. Takei, M. Nonogi, A. Hibara, T. Kitamori, and H. B. Kim, "Tuning microchannel wettability and fabrication of multiplestep Laplace valves," Lab on a Chip, vol. 7, no. 5, pp. 596-602, 2007.

[13] S. Shoji and K. Kawai, "Flow control methods and devices in micrometer scale channels," Topics in Current Chemistry, vol. 304, pp. 1-25, 2011.

[14] H. Sugino, K. Ozaki, Y. Shirasaki, T. Arakawa, S. Shoji, and T. Funatsu, "On-chip microfluidic sorting with fluorescence spectrum detection and multiway separation," Lab on a Chip, vol. 9, no. 9, pp. 1254-1260, 2009.

[15] Y. Shirasaki, J. Tanaka, H. Makazu et al., "On-chip cell sorting system using laser-induced heating of a thermoreversible gelation polymer to control flow," Analytical Chemistry, vol. 78, no. 3, pp. 695-701, 2006.

[16] H. Sugino, T. Arakawa, Y. Nara et al., "Integration in a multilayer microfluidic chip of 8 parallel cell sorters with flow control by sol-gel transition of thermoreversible gelation polymer," Lab on a Chip, vol. 10, no. 19, pp. 2559-2565, 2010.
[17] S. Y. Lee, Y. Lee, J. E. Kim, T. G. Park, and C. H. Ahn, "A novel pH-sensitive PEG-PPG-PEG copolymer displaying a closedloop sol-gel-sol transition," Journal of Materials Chemistry, vol. 19, no. 43, pp. 8198-8201, 2009. 

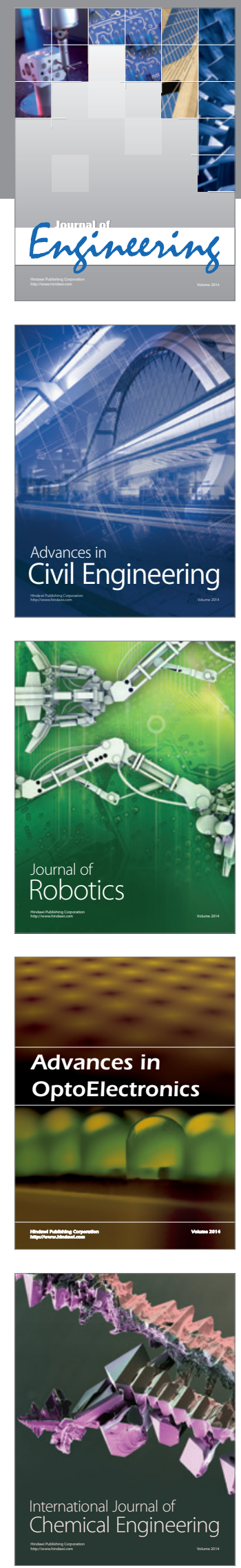

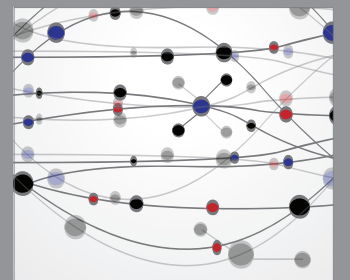

The Scientific World Journal
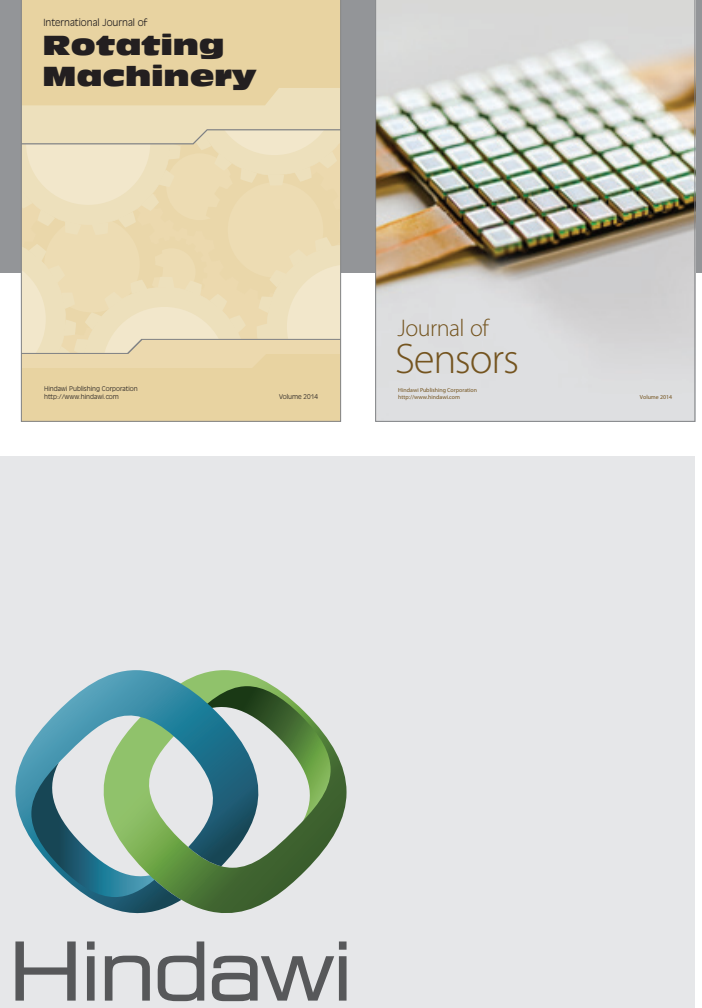

Submit your manuscripts at http://www.hindawi.com
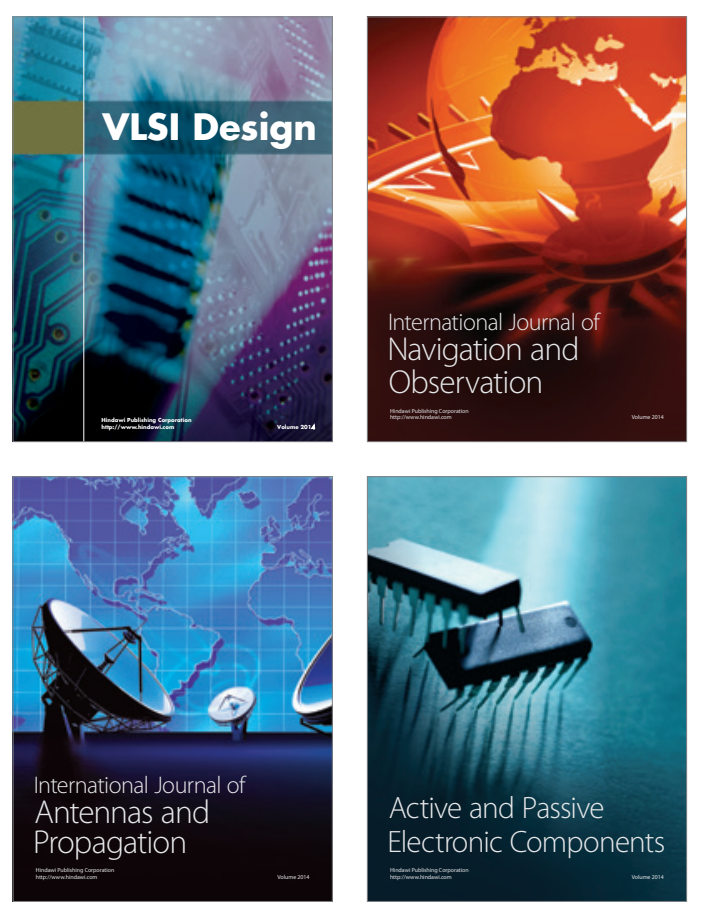
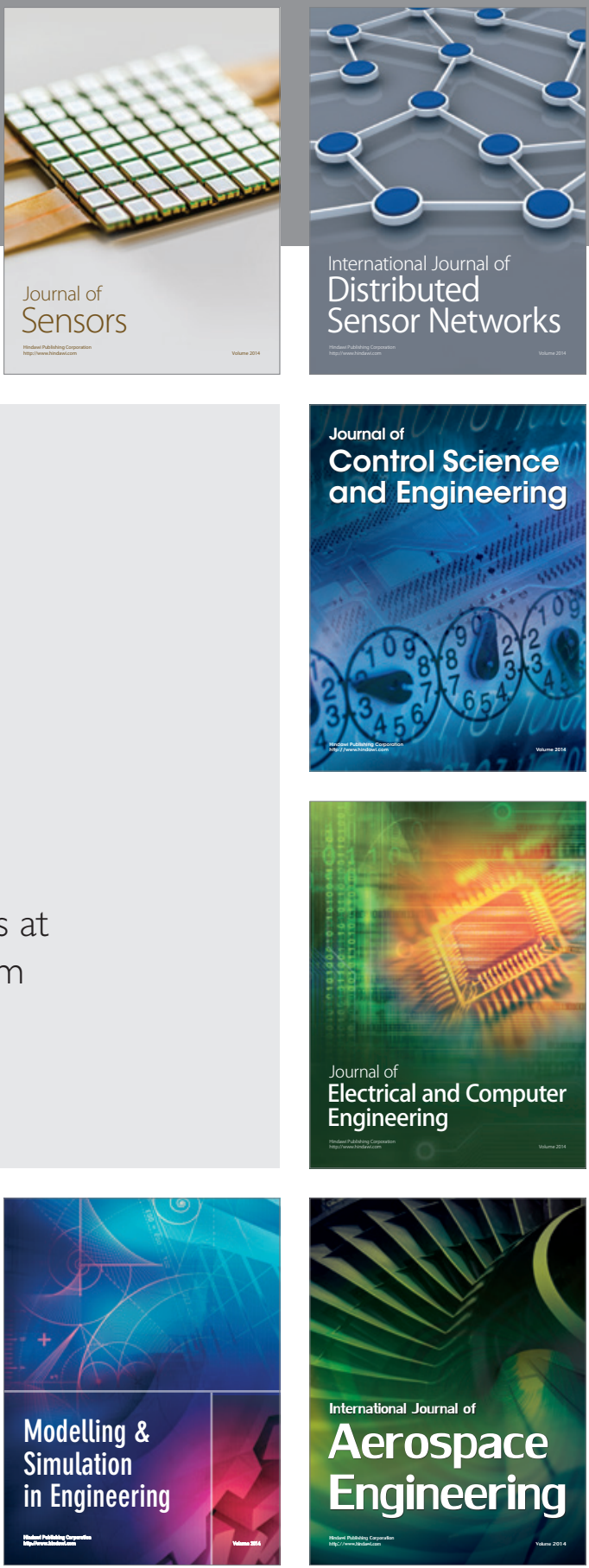

Journal of

Control Science

and Engineering
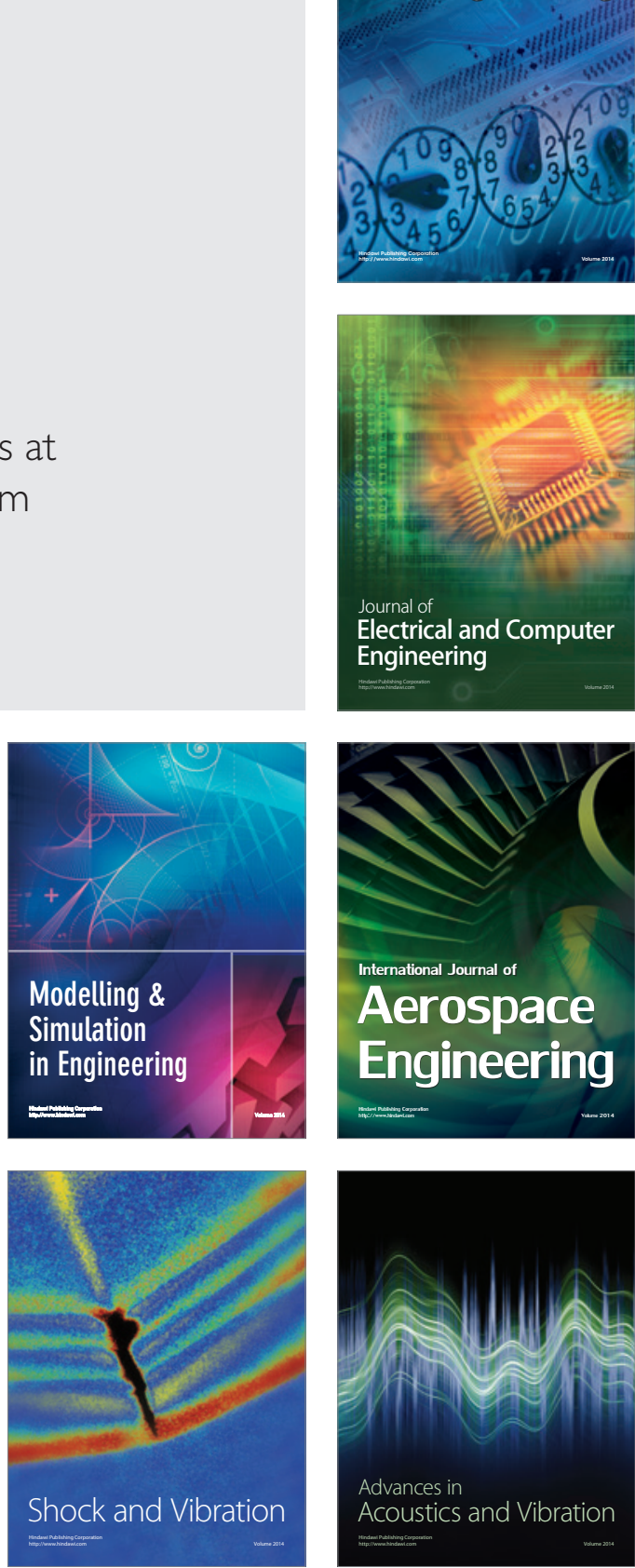